\title{
PREDICTORS OF CANDIDA BLOOD STEAM INECTION IN NEWBORNS ADMITTED IN LEVEL III NEONATAL UNIT NEW DELHI
}

A.Kumar*, S. Namdev*, A. Mahor ** V.S.Randhawa** S. Nangia, ${ }^{*}$ A.Saili* From Departments of Neonatology ${ }^{*}$ and Microbiology ${ }^{* *}$ Lady Hardinge Medical College and Associated Kalawati Saran Children Hospital New Delhi

\section{Background}

Candida is one of the leading causes of blood stream infections (BSI) in neonatal intensive care units (NICUs) and is associated with high morbidity and mortality

The gross mortality of fungal infections in NICUs ranges from $25 \%$ to $50^{1,2}$ While in an Indian study, Candida attributed deaths occurred in $17 \%$ cases $^{3}$

The clinical presentation of invasive fungal and bacterial infection is similar, which may cause diagnostic and treatment delay

Given the high mortality and difficulty in establishing an early diagnosis, it is important to determine risk factors/predictors for Candida BSI

\section{Objective}

To find the risk factors/predictors for Candida BSI in newborns by comparing them with newborns with Klebsiella BSI

\section{Methods}

Retrospective Case Control Study

Period between January 2014 and December 2017 (48 months)

Level III NICU in New Delhi

Study population included babies who grew Candida in blood culture

Data on patient demographics, underlying diseases, medications, central catheters, nutrition, ventilator use etc. was retrieved

Risk factors were evaluated from the time of admission until the onset of
Candida BSI and then till discharge or death. For each case, one neonate who grew Klebsiella on blood culture was included

Data analysis was performed using SPSS Version 20.0. Risk factors were assessed using Univariate analysis All $p$ values were two tailed and values $<.05$ were taken as significant

\section{Results}

Thirty out of total of 8928 admitted newborns developed Candida BSI (3.36 per 1000)

Examination of a standard battery of conditions associated with morbidity in both groups e.g. birth-weight, gestation, asphyxia, ventilation,

Incidence of Candida BSI

(January- 2014 to December 2017)

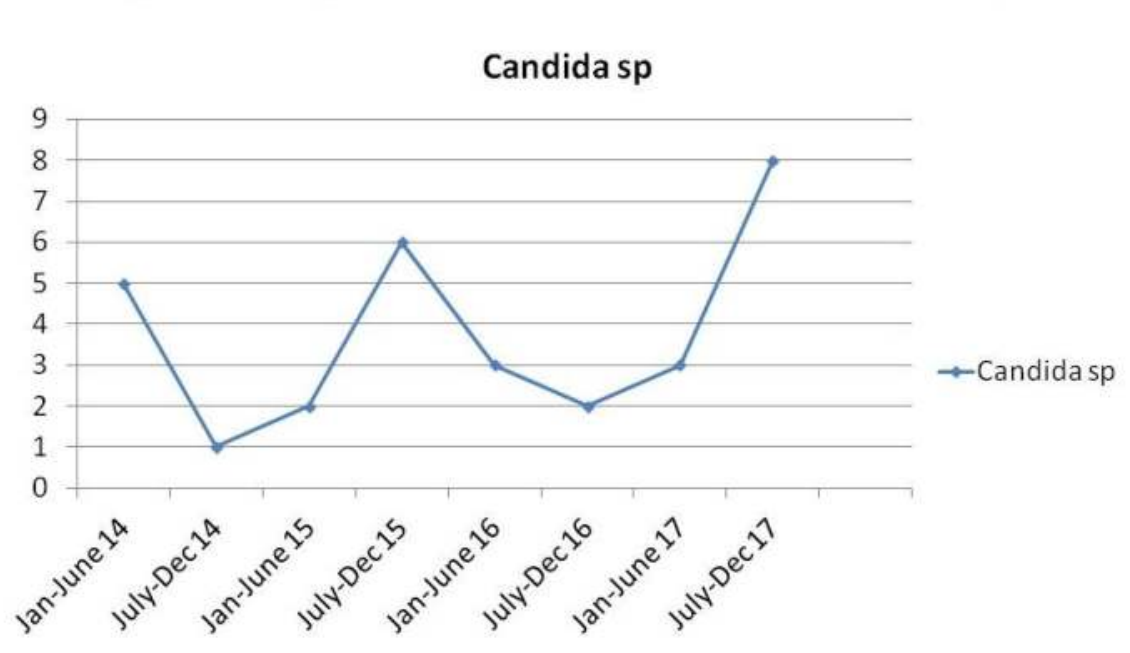

Risk Factors for Candida BSI

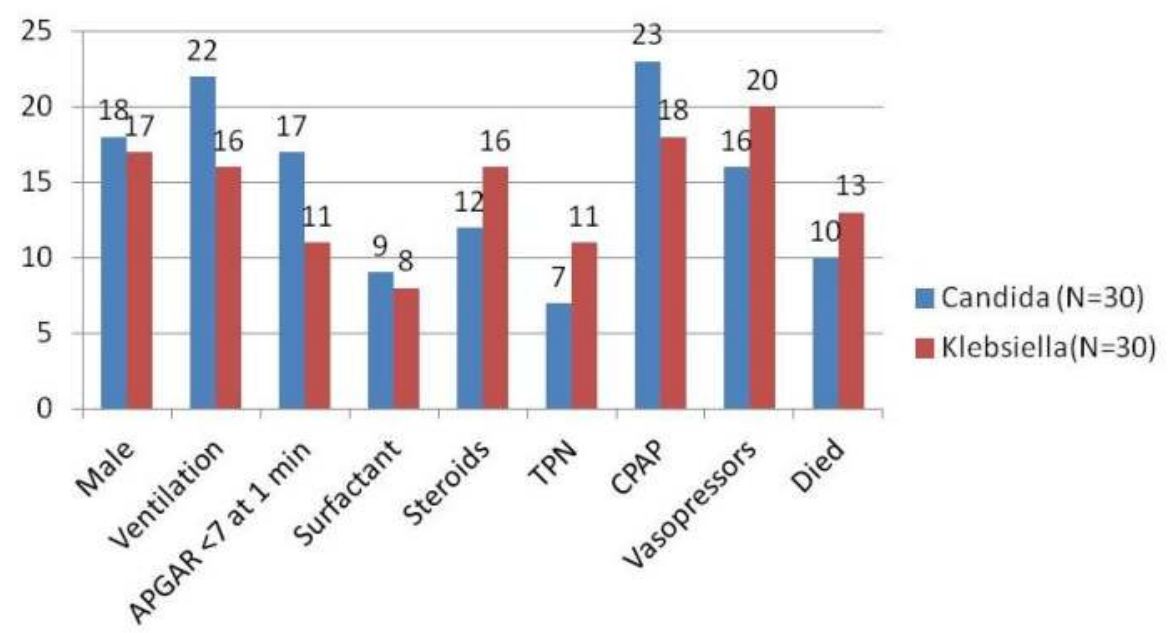

Table 1. Univariate analysis

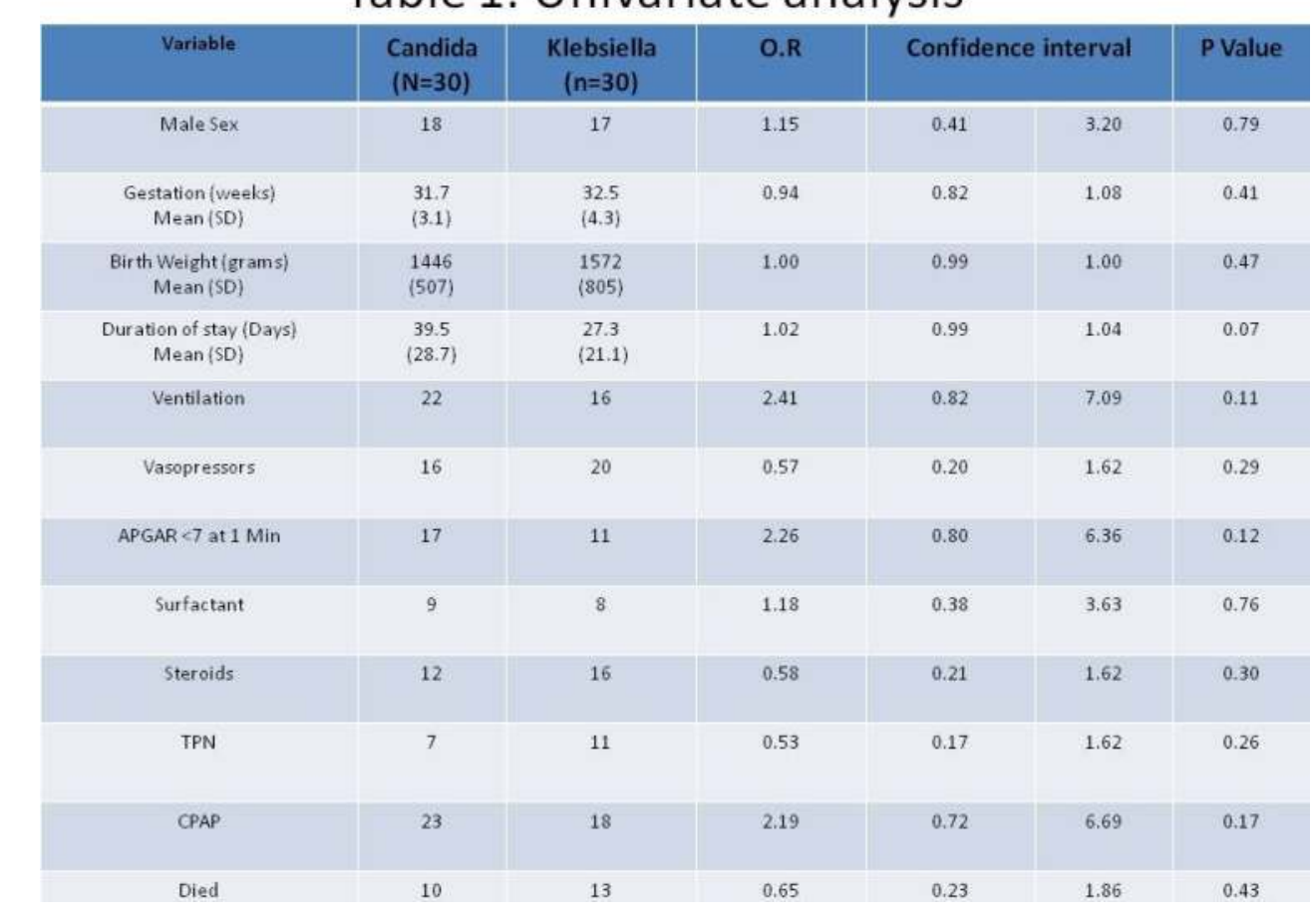

vasopressors use, surfactant, steroid, parenteral nutrition etc did not reveal any notable differences

Candida BSI and Klebsiella BSI were associated with similar mortality (33\% vs. $43 \% \quad P>0.05$ )

\section{Discussion}

Several factors have been identified as contributing to an increased incidence of neonatal candidemia, including prolonged endotracheal intubation, indwelling catheterization, parenteral nutrition, broad-spectrum antibiotics, \& prolonged antibiotic therapy duration 4,5

Similarly antenatal steroid use, cesarean section, male gender, low birth weight, parenteral nutrition, percutaneous and umbilical catheter insertion, mechanical ventilation and low Apgar scores were found to be risk factors for bacterial blood stream infections $^{6}$. We found no statistical difference between risk factors predisposing to Candida BSI or Klebsiella BSI
In a study from Kuwait, the case-fatality rate for Candida species in LOS was $22 \%$, significantly higher than the casefatality rate of Gram-negative bacillary infections, or any other type of infection $^{7}$

In contrast in our study we found comparable mortality (33\% vs. $43 \%$ $\mathrm{P}>0.05$ ) among newborns with Candida BSI and Klebsiella BSI

\section{Limitations}

Retrospective Study

We were not able to assess all variables Limited by the completeness of documentation by the treating neonatologist

Study period and sample size was small Results could be NICU specific

\section{Conclusions}

Newborns admitted with Candida or Klebsiella BSI in level III neonatal units have similar epidemiological characteristics, morbidity and mortality

\section{References}

1. Weese-Mayer DE, Fondriest DW, Brouillette RT, Shulman ST. Pediatr Infect Dis J 1987;6:190-6.

2. Wadile RG, Bhate VM. Indian J Pathol Microbiol 2015;58:472-74.

3. Narang A, Agrawal PB, Chakrabarti A, Kumar P. J Trop Pediatr 1998;44:104-8.

Singhi S, Rao DS, Chakrabarti A. Pediatr Crit Care Med 2008;9:91-5.

5. Chen J, Jiang Y, Wei B, et al. BMC Infectious Diseases 2016;16: 700

6. Bolat F, Uslu S, Bolat G, et al. Indian Pediatr 2012;49:951-7.

7. Hammoud MS, Al-Taiar A, Thalib L, et al. $J$ Paediatr Child Health 2012;48:604-9. 